\title{
The complementary graphene growth and etching revealed by large-scale kinetic Monte Carlo simulation
}

\author{
Xiao Kong $\mathbb{1}^{1}$, Jianing Zhuang ${ }^{2}$, Liyan Zhu $\mathbb{B}^{1,3}$ and Feng Ding $\mathbb{\mathbb { D }}^{1,2,4 凶}$
}

To fully understand the kinetics of graphene growth, large-scale atomic simulations of graphene islands evolution up to macro sizes (i.e., graphene islands of a few micrometers or with billions of carbon atoms) during growth and etching is essential, but remains a great challenge. In this paper, we developed a low computational cost large-scale kinetic Monte Carlo (KMC) algorithm, which includes all possible events of carbon attachments and detachments on various edge sites of graphene islands. Such a method allows us to simulate the evolution of graphene islands with sizes up to tens of micrometers during either growth or etching with a single CPU core. With this approach and the carefully fitted parameters, we have reproduced the experimentally observed evolution of graphene islands during both growth or etching on $\mathrm{Pt}(111)$ surface, and revealed more atomic details of graphene growth and etching. Based on the atomic simulations, we discovered a complementary relationship of graphene growth and etching-the route of graphene island shape evolution during growth is exactly the same as that of the etching of a hole in graphene and that of graphene island etching is exactly same as that of hole growth. The complementary relation brings us a basic principle to understand the growth and etching of graphene, and other 2D materials from atomic scale to macro size and the KMC algorithm is expected to be further developed into a standard simulation package for investigating the growth mechanism of 2D materials on various substrates.

npj Computational Materials (2021)7:14; https://doi.org/10.1038/s41524-020-00489-y

\section{INTRODUCTION}

Graphene is a two-dimensional carbon allotrope that was firstly isolated in 2004 by Geim and Novoselov and their colleagues, and then has attracted intensive studies for $>15$ years ${ }^{1}$. Monocrystalline graphene has excellent properties, such as very high mechanical strength, thermal conductivity, carrier mobility and emergent Dirac excitations, and many consequent potential applications $^{2-6}$. To realize the vast applications of graphene, a mass of physical and chemical methods, such as mechanical and chemical exfoliation ${ }^{7-10}$, epitaxial growth ${ }^{11-13}$, thermal decomposition of silicon carbide ${ }^{14-16}$ and chemical vapor deposition (CVD) ${ }^{17-37}$, was developed to synthesize various graphene samples. Among them, the CVD method is the only one that enables the production of high-quality single-layer graphene films in macro sizes, such as the wafer-scale single-crystalline graphene grown on $\mathrm{Cu}$ surface. In real experiments, by tuning the experimental parameters, such as concentrations of carbon precursor and carrier gases, temperature, substrate type, and morphology, graphene islands of various shapes have been broadly observed ${ }^{30-37}$. At atomic scale, graphene CVD growth involves many physical and chemical steps, such as feedstock molecules decomposition, precursor diffusion on the substrate, carbon attachment, and detachment at the graphene edge. To realize the controllable synthesis, it is crucial to understand the mechanism of graphene CVD growth, and identify the threshold step and the key factors that control the growth ${ }^{38-48}$. Thus, the large-scale atomic simulation of graphene growth is highly desirable, and, besides graphene, such a simulation method is also urgently needed for understanding the growth mechanism of various $2 \mathrm{D}$ materials and their controllable synthesis.
Theoretically, the kinetics of graphene growth or etching has been understood based on the theory of kink nucleation and propagation ${ }^{34,38}$, in which kink nucleation at an armchair (AC) or zigzag ( $Z Z$ ) edge is considered as the threshold step of edge propagation, and the existing kinks propagate rather fast. Based on this theory, the growth rate of any graphene edge can be estimated, and the graphene shape evolution during growth or etching can be predicted by applying the theory of kinetic Wulff construction. Such theory has been applied to explain and predict the graphene morphology evolution during CVD growth or etching and qualitative agreements with experimental observations were broadly reported ${ }^{33,34}$. While, to explore more atomic details during graphene growth or etching, such as the effect of concentration gradient of carbon precursors on the substrate, the effect of substrate inhomogeneity, the lattice mismatch between graphene and the substrate, the previous theory must be extensively modified, and many of these effects can only be revealed by atomic simulations.

Phase field theory allows us to explore the overall shape evolution of an island of graphene or other $2 \mathrm{D}$ materials on various substrates with a concentration gradient ${ }^{36,37,49-52}$. But it ignored the atomic structure details in the simulations. Molecular dynamic simulation includes all the atomic details, but the very short simulation time mostly leads to defective graphene islands of a few nm or less and, thus, it cannot offer a complete scenery of graphene growth from the nucleation of a nanometer-sized island to a macro-sized graphene film ${ }^{53}$. Recently, some kinetic Monte Carlo (KMC) methods were developed to explore the growth and etching of graphene and other 2D materials, but the maxim sizes of the simulated islands of graphene or 2D materials were generally $<10 \mathrm{~nm}$ (refs. ${ }^{54-57}$ ), which was far from sufficient to

\footnotetext{
${ }^{1}$ Centre for Multidimensional Carbon Materials, Institute for Basic Science, Ulsan 44919, Korea. ${ }^{2}$ Institute of Textiles and Clothing, Hong Kong Polytechnic University, 999077 Kowloon Hong Kong, China. ${ }^{3}$ Department of Physics, and Jiangsu Key Laboratory of Modern Measurement Technology and Intelligent Systems, Huaiyin Normal University,

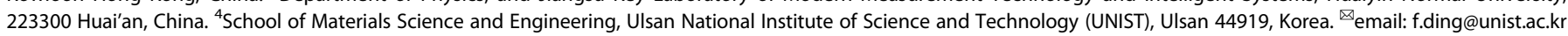


(a)

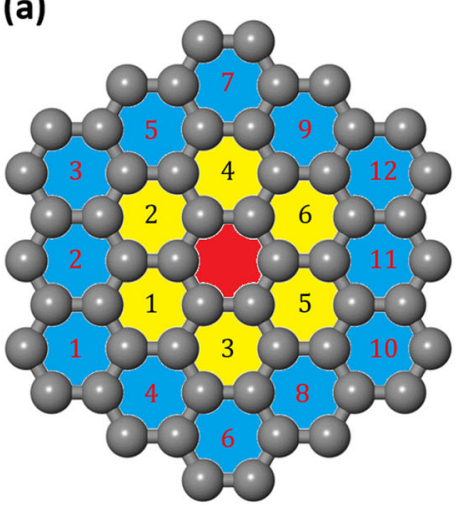

(b)
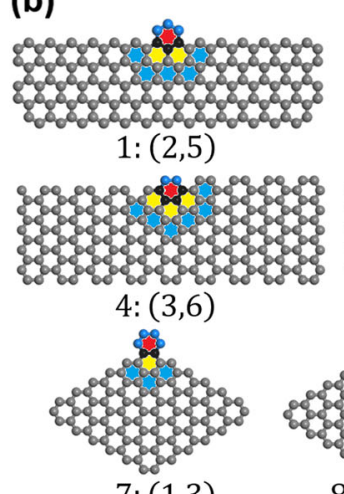

$7:(1,3)$

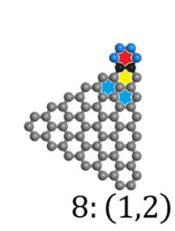

$5:(3,5)$

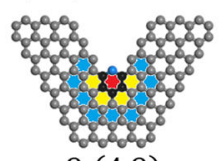

$9:(4,9)$

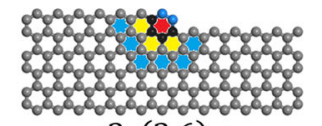

3: $(3,6)$

2: $(4,7)$

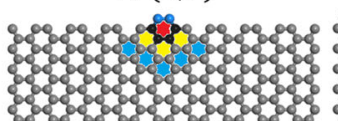

6.
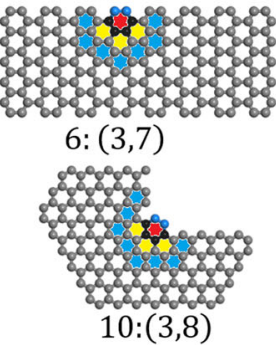

Fig. 1 Structure of a local site in a graphene island and some key events used in the KMC method. a Six nearest neighbors (yellow) and 12 second-nearest neighbors (blue) of a hexagon in a graphene lattice. b Representative events (red) in graphene growth or etching with their corresponding indexes $(m, n)$ shown below each event. The blue atoms are those to be added or removed during the event of adding or removal. 1 and 2 are nucleation at zigzag edge during growth and etching, respectively; 3 and 4 are kink propagation at the ZZ and AC edges, respectively; 5 and 6 are armchair nucleation at an armchair edge during growth and etching, respectively; 7 and 8 are events at a convex corner; and 9 and 10 are events at a concave corner, respectively.

reveal the size-dependent growth behavior or graphene, such as the fractal-shaped graphene islands grown with a precursor concentration gradient on the substrate surface.

In this paper, we present a large-scale KMC method for the simulation of graphene growth/etching on a transition metal surface. The order- $\mathrm{N}$ algorithm allows us to explore the graphene growth up to the size of tens of $\mu \mathrm{m}$ with a single CPU core, and to reveal the morphology evolution of a graphene island as the dimension crossing many orders of magnitude, from a single hexagon to that with billions of atoms. With this simulation package, we have reproduced the experimentally observed shape evolution of graphene and holes in graphene on Pt surface, during both growth and etching processes, and insights into the mechanism of graphene growth on Pt has been achieved. Our study reveals a very important complementary principle of graphene growth and etching. Besides, our algorithm is efficient in the scale of experimental observations and each event has a clear physical meaning, which are very beneficial for understanding the mechanisms of the growth and etching of 2D materials precisely. Therefore, it is expected to be a general method for the simulation of various $2 \mathrm{D}$ materials.

\section{RESULTS AND DISCUSSION \\ Algorithm of large-scale kinetic Monte Carlo simulation}

The framework of our method is same with general KMC algorithm (see Supplementary Material for the process of general KMC and our method). Here, we briefly introduce the key events of our simulation, the calculation of the corresponding energy barriers, and the algorithm for fast simulation.

The initial configuration is a graphene island with certain number of hexagons, and the events are defined as adding or removing one hexagon to or from an edge of the graphene island. We used 0 or 1 to record the existences of nearest and secondnearest hexagons, and the numbers of nearest and secondnearest hexagons, $(m, n)$, around the active hexagon to characterize the type of the event, as shown in Fig. 1a. The number of nearest hexagons, $m$, could be an integer from 1 to 4 since $m=0$ corresponded a nucleation event, which is not considered in this study and $m=5$ or 6 corresponded an existing hexagon in the central of graphene lattice and was hardly to be removed. Likewise, the number of second-nearest hexagons, $n$, ranged from 1 to 10 . According to the kink nucleation and propagation theory, three kind of events are critical for graphene growth or etching, which are kink nucleation at a ZZ edge, kink nucleation at an AC edge, and kink propagation. Besides the four events on straight $Z Z$ and $A C$ edges, adding and removing hexagons at various types of convex and concave sites of a graphene island must be considered and, in general, adding (removing) a hexagon at a concave (convex) site is energetically more favorable than that at a straight edge, while adding (removing) a hexagon at a convex (concave) site is energetically less favorable than that at a straight edge. Some typical types of events were shown in Fig. 1b, and more types of events were listed in the Supplementary Materials, Supplementary Fig. 2.

Each unit of a hexagon graphene lattice has two carbon atoms and thus there are two steps of forming a new hexagon, one of them corresponds an intermediate state and another one is the global minimum. The events of adding or removing a hexagon should correspond the global minimum and the barrier of the event should be the highest energy of the whole process. This ensures the correct kinetics of adding/removing carbon to/from a graphene edge. The Gibbs free-energy barrier of adding a new hexagon at the site of $(m, n)$ is

$G_{\mathrm{b}+}^{(m, n)}=E_{\mathrm{b}}^{(m, n)}-n_{\mathrm{c}} \mu$,

where $E_{\mathrm{b}}^{(m, n)}$ is the barrier of the reaction, $\mu$ is the chemical potential of a carbon atom, which is a function of the concentration of carbon precursor on the substrate, and $n_{C}$ is the number of carbon atoms required to form a new hexagon at the $(m, n)$ site. Consequently, the removal of such a hexagon has a barrier of

$G_{\mathrm{b}-}^{(m, n)}=E_{\mathrm{b}}^{(m, n)}-\Delta E^{(m, n)}+n_{\mathrm{c}} \mu$,

where $\Delta E^{(m, n)}$ is the energy difference between the final and initial states or the reaction energy. As an example, we consider the graphene growth on a Pt(111) surface. We calculated the reaction energies of key events during graphene growth and etching on the substrate by density-functional theory (DFT). The results of ZZ edge nucleation, $A C$ edge nucleation, $120^{\circ}$ convex corner growth, and $60^{\circ}$ concave corner growth are $\Delta E^{(2,5)}=2.48 \mathrm{eV}, \Delta E^{(3,5)}=1.19$ $\mathrm{eV}, \Delta E^{(1,3)}=3.09 \mathrm{eV}$, and $\Delta E^{(4,9)}=-1.95 \mathrm{eV}$, respectively. The order of these reaction energies agrees well with our intuition that adding precursors to a concave (convex) site is the easiest (hardest) and the kink nucleation on a ZZ edge is more difficult than that on an $A C$ edge $e^{34,38}$. Besides, the consideration of the reverse processes ensures correct thermodynamics of the system during the evolution. In Fig. 2a, we listed these calculated events and their reverse events with their indexes, barriers, structure sketches, and reaction energy profiles in a table. 
(a)

\begin{tabular}{|c|c|c|c|c|}
\hline $\begin{array}{l}\text { ZZ edge nucleation } \\
(2,5)\end{array}$ & $\begin{array}{l}\text { F: } E_{\mathrm{b}}^{(2,5)}-3 \mu \\
\text { R: } E_{\mathrm{b}}^{(2,5)}-\Delta E^{(2,5)}+3 \mu\end{array}$ & \multirow{2}{*}{$\begin{array}{l}\Delta E^{(2,5)}=2.48 \mathrm{eV} \\
E_{\mathrm{b}}^{(2,5)}=2.98 \mathrm{eV}\end{array}$} & $\Rightarrow$ & 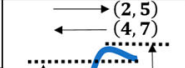 \\
\hline $\begin{array}{l}\text { ZZ edge completion } \\
(4,7)\end{array}$ & $\begin{array}{l}\mathrm{F}: E_{\mathrm{b}}^{(2,5)}-\Delta E^{(2,5)}-\mu \\
\mathrm{R}: E_{\mathrm{b}}^{(2,5)}+\mu\end{array}$ & & & $\begin{array}{c}\Delta E \\
\ldots 7 . \ldots \ldots \ldots \ldots . .\end{array}$ \\
\hline $\begin{array}{l}\text { AC edge nucleation } \\
(3,5)\end{array}$ & $\begin{array}{l}\mathrm{F}: E_{\mathrm{b}}^{(3,5)}-2 \mu \\
\mathrm{R}: E_{\mathrm{b}}^{(3,5)}-\Delta E^{(3,5)}+2 \mu\end{array}$ & \multirow{2}{*}{$\begin{array}{l}\Delta E^{(3,5)}=1.19 \mathrm{eV} \\
E_{\mathrm{b}}^{(3,5)}=1.69 \mathrm{eV}\end{array}$} & & $\begin{array}{l}\longrightarrow(3,5) \\
\longleftarrow(3,7)\end{array}$ \\
\hline $\begin{array}{l}\text { AC edge completion } \\
(3,7)\end{array}$ & $\begin{array}{l}\mathrm{F}: E_{\mathrm{b}}^{(3,5)}-\Delta E^{(3,5)}-2 \mu \\
\mathrm{R}: E_{\mathrm{b}}^{(3,5)}+2 \mu\end{array}$ & & & $\Delta E E_{\mathrm{b}}$ \\
\hline $\begin{array}{l}\text { Kink propagation } \\
(3,6)\end{array}$ & $\begin{array}{l}\mathrm{F}: E_{\mathrm{b}}^{(3,6)}-2 \mu \\
\mathrm{R}: E_{\mathrm{b}}^{(3,6)}+2 \mu\end{array}$ & $\begin{array}{l}\Delta E^{(3,6)}=0 \mathrm{eV} \\
E_{\mathrm{b}}^{(3,6)}=0.5 \mathrm{eV}\end{array}$ & & $E_{\mathrm{b}}$ \\
\hline $\begin{array}{l}\text { Convex corner growth } \\
(1,3)\end{array}$ & $\begin{array}{l}\mathrm{F}: E_{\mathrm{b}}^{(1,3)}-4 \mu \\
\mathrm{R}: E_{\mathrm{b}}^{(1,3)}-\Delta E^{(1,3)}+4 \mu\end{array}$ & $\begin{array}{l}\Delta E^{(1,3)}=3.09 \mathrm{eV} \\
E_{\mathrm{b}}^{(1,3)}=3.59 \mathrm{eV}\end{array}$ & 080 & $\Delta E$ \\
\hline $\begin{array}{l}\text { Concave corner growth } \\
(4,9)\end{array}$ & $\begin{array}{l}\mathrm{F}: E_{\mathrm{b}}^{(4,9)}+\mu \\
\mathrm{R}: E_{\mathrm{b}}^{(4,9)}-\Delta E^{(4,9)}-\mu\end{array}$ & $\begin{array}{l}\Delta E^{(4,9)}=-1.95 \mathrm{eV} \\
E_{\mathrm{b}}^{(4,9)}=0.5 \mathrm{eV}\end{array}$ & & \begin{tabular}{c}
$E_{\mathrm{b}}$ \\
\hdashline$E$
\end{tabular} \\
\hline
\end{tabular}

(b) 4

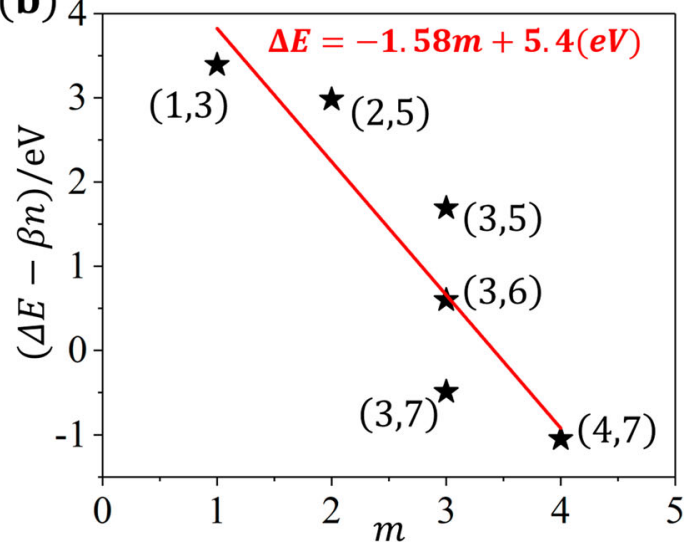

Fig. 2 Key events and corresponding reaction barriers. a List of some key events and the calculated reaction energies and the estimated reaction barriers. The first column lists the types of atomic steps and corresponding forward event indices $(m, n)$. The second column is Gibbs free-energy barrier of forward (F) and reverse (R) reactions. In the third column, we list the calculated reaction energy and estimated barriers of these events on a Pt(111) surface. In the fourth column, we show local structure of these events, where blue and red arrows indicate forward and reverse reactions, respectively. In the fifth column, we sketch the reactions, where the reaction energies $\Delta E$ and barriers $E_{\mathrm{b}}$ of them are also shown. b Linear fitting of reaction energy (after taking $\beta=0.1 \mathrm{eV}$ ) with number of nearest neighbors $m$.

To complete the reaction energy list for all considered events, such as adding or removal a hexagon with the index of $(2,7),(4,3)$ $\ldots$, we construct a formula to fit their reaction energies as:

$\Delta E^{(m, n)}=a m+\beta n+\Delta E^{(0)}$,

where $\Delta E^{(0)}=5.4 \mathrm{eV}$ is a constant, fitting parameter $a=-1.58 \mathrm{eV}$ represents the interaction between the hexagon and its first nearest neighbors and $\beta=-0.1 \mathrm{eV}$ represent the interaction of the hexagon and its second-nearest neighbors. Besides, following cases were considered in the fitting:

(i) The reaction energy of kink propagation $(3,6)$ must be $0 \mathrm{eV}$, $\Delta E^{(3,6)}=0$, because the local structure was not changed by the reaction.

(ii) The reaction energy of annihilating two kinks is the negative of nucleating two kinks. So, we have $\Delta E^{(4,7)}=-\Delta E^{(2,5)}$ for $\mathrm{ZZ}$ edge and $\Delta E^{(3,7)}=-\Delta E^{(3,5)}$ for $\mathrm{AC}$ edge.

(iii) The nucleation energies on an $A C$-like edge, such as $(3,3)$, $(3,4),(3,8)$, and $(3,9) \ldots$, have large errors from the DFT calculated event, $A C$ nucleation $(3,5)$. So, we reconsidered these events based on the DFT result (see SI).

It is important to note that the simulated results are generally very similar in quite large ranges of these parameters and, therefore, the insights of graphene growth revealed by the KMC simulation are robust.

Next, let's consider the difference between the reaction energy and the barrier of an event, $E_{\mathrm{b}}^{(m, n)}-\Delta E^{(m, n)}$. Generally, the barrier of a reaction highly depends on the initial, final configurations, the intermediate states, and the specific configuration of the graphene site on the catalyst surface and its precisely calculation is very challenging. In a recent calculation, Wang et al., proved that the barrier of a nucleation event can be estimated by summation of the formation energy of the nucleus and the barrier of kink propagation ${ }^{58}$. So, we consider the energy difference between a transition state and the initial or final state, whichever is higher, is a constant, $0.5 \mathrm{eV}$. Such a constant energy barrier for all events keeps the central idea of the theory of kink nucleation and propagation, and the exact value will not have much impact on the evolution of graphene because the probabilities of most events are changed by a same factor.

The list of energy barriers can be used to simulate the graphene growth or etching in a homogeneous environment. In this paper, we focus on studying the general behaviors of graphene growth and etching in CVD experiments. Here, we assumed that the concentration of carbon adatoms on the whole substrate is a constant, which is applicable for the attachment limit of graphene growth $^{35-37,49}$. The lattice mismatch between graphene and substrate is one of the key factors in understanding growth mechanism of graphene. The effect of substrate contains several parts. Firstly, the activity of substrate determines the bonding between the graphene edge and substrate, and therefore determines the reaction energy of different events, this part has been considered in our calculations. Secondly, the reaction energy of an event is a function of the relative coordinates on substrate lattice, which may lead to the change of growth mode from kink nucleation and propagation (on less active and lattice matched substrates) to growth in unit of Moire pattern (on more active and lattice mismatched substrates $)^{59}$. To fully understand the effect of substrate is our ultimate goal, but tremendous further efforts are required to obtain the functions of these events on a specific substrate $^{60}$. In this manuscript, the fluctuation of van der Waals (VDW) interaction from the lattice mismatch between graphene and the substrate is neglected. As we discussed in the introduction, these effects can be further explored by modifying the barriers of these events as functions of the local environment.

We further optimized the algorithm to simulate the growth or etching of very large graphene islands. Firstly, we ignore the bulk part of a graphene island and only recorded the hexagons of its edge because the bulk part is highly robust, and all the events of adding new hexagons or hexagonal removal occurs on the edge. Secondly, after a reaction, we only update very few events of the event list occurred in the vicinal of the added or removed hexagon. These approaches, together with applying advanced computational algorithms, greatly reduced the computation cost, and allow us to simulate graphene islands with billions of hexagons or with lateral size up to tens of micrometers.

\section{KMC simulations of graphene on $\mathrm{Pt}(111)$ surface}

With the developed KMC algorithm and fitted parameters, now let's simulate the evolution of graphene islands and holes in graphene on $\mathrm{Pt}(111)$ surface during both growth and etching processes, respectively. Such processes have been systematically studied in experiments and the theory of kink nucleation and propagation has been applied to explain the experimentally observed graphene islands and the holes in graphene ${ }^{34}$. It was found that a growing graphene island always presents a hexagonal shape with six straight $\mathrm{ZZ}$ edges and six sharp convex corners, while an etched graphene island will finally reach a steady 
(a)
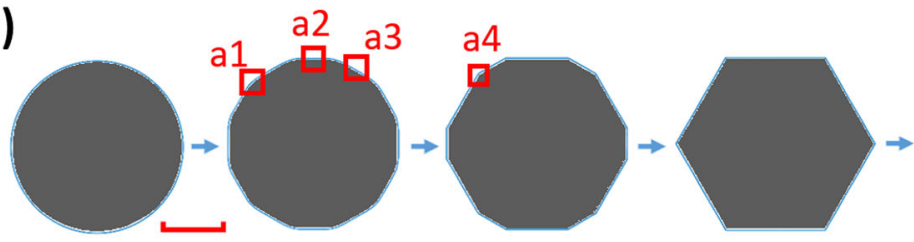

(1): $t=0$

(2): $6.96 \times 10^{4}$

(3): $1.1 \times 10^{6}$

(4): $2.64 \times 10^{7}$

(5): $9.66 \times 10^{8}$

(b)
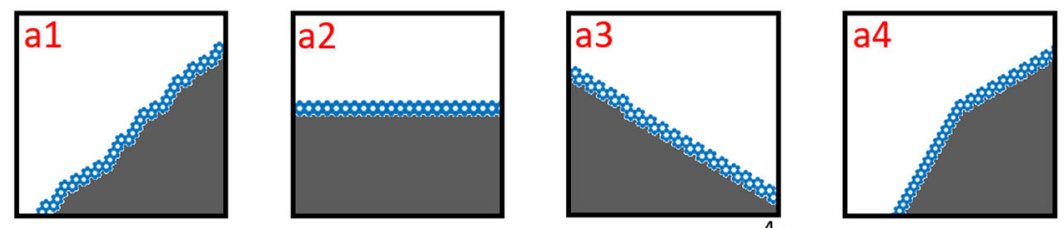

(c)

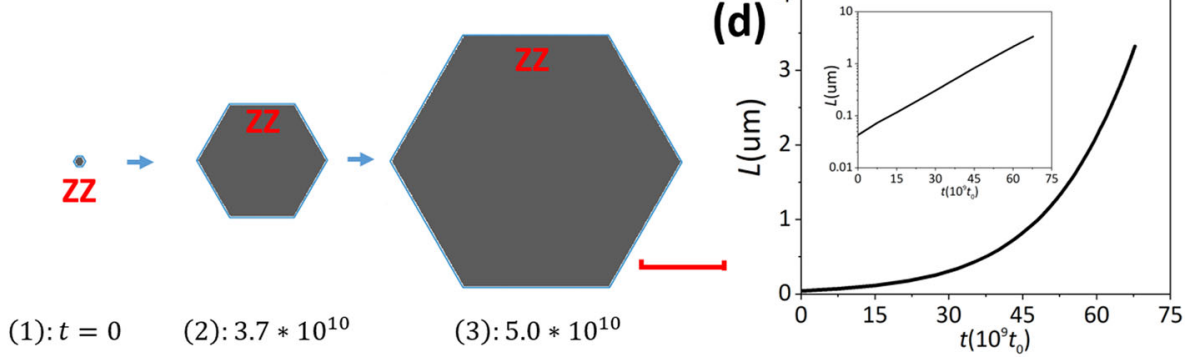

Fig. 3 Growth simulation of a graphene island. The simulation condition is $\mu=0.2 \mathrm{eV}$ and $k_{\mathrm{B}} T=0.1 \mathrm{eV}$. a Representative snapshots of a trajectory of KMC simulation for graphene growth. The evolution time is labeled below each snapshot in the unit of $t_{0}$, where $t_{0}=h / k_{\mathrm{B}} T$. The scale bar is $2.0 \mu \mathrm{m}$ for all snapshots. b Atomic edge structures in simulation (a), a1 is a rounded corner near the crossover region from zigzag edge to armchair edge during the initial stage of the evolution, a2 is a straight zigzag edge, a3 is a straight armchair edge, and a4 is a sharp corner between a zigzag edge and an armchair edge, respectively. c Representative snapshots of a growth simulation of zigzag graphene island. The evolution time is labeled below each snapshot in the unit of $t_{0}$, where $t_{0}=h / k_{\mathrm{B}} T$. The scale bar is $1.0 \mu \mathrm{m}$ for all snapshots. $\mathbf{d}$ Plot of the island size in c with growth time by linear and logarithmic scale (inset).

state with a dodecagon shape with $1219^{\circ}$ edges. In contrast, a hole in graphene presents straight ZZ edges and sharp concave corners during etching, but its circumference generally becomes rounded during growth. In order to verify the simulation method and reveal further details of graphene growth and etching on $\mathrm{Pt}$ (111) surface, we will explore this system with the developed KMC method.

We firstly investigated the growth of graphene. The growth rate of an edge $X$ can be approximatively expressed $a s^{38,61}$

$v(X) \propto s_{K}(X)+s_{A}(X) \mathrm{e}^{-\frac{G_{b+}^{(3,5)}-G_{b+}^{(3,6)}}{k_{B} T}}+s_{Z}(X) \mathrm{e}^{-\frac{G_{b+}^{(2,5)}-G_{b+}^{(3,6)}}{k_{B} T}}$,

where $s_{K}(X), s_{A}(X)$, and $s_{Z}(X)$ are concentrations of kink, $A C$ and $Z Z$ sites of the edge, respectively, $G_{\mathrm{b}+}^{(2,5)}, G_{\mathrm{b}+}^{(3,5)}$, and $G_{\mathrm{b}+}^{(3,6)}$ are the freeenergy barriers of adding a hexagon to a ZZ edge, an AC edge, and a kink site, respectively. Since in generally, we have $\mathrm{e}^{-\frac{G_{\mathbf{b}+}^{(3,5)}-G_{\mathbf{b}+}^{(3,6)}}{k_{\mathrm{B}} T}} \ll 1$ and $\mathrm{e}^{-\frac{G_{\mathrm{b}+}^{(2,5)}-G_{\mathbf{b}+}^{(3,6)}}{k_{\mathrm{B}} T}} \ll 1$, the propagation rate is approximately proportional to $s_{\mathrm{K}}(X)$ or the kink concentration of the graphene edge. Among all possible graphene edges, the $19^{\circ}$ edges are the edges with the highest kink concentration, and thus generally are the edges that propagate fastest ${ }^{34,38}$.

According to the theory of kinetic Wulff construction, the shape of a graphene island during growth is determined by the slowest propagating edges, which always leads to hexagonal graphene island with straight $\mathrm{ZZ}$ edges. We performed our method in simulating the growth behaviors of graphene islands, the KMC condition was $\mu=0.2 \mathrm{eV}$ and $k_{\mathrm{B}} T=0.1 \mathrm{eV}$. Figure $3 a$ presents the evolution of a graphene island during a KMC simulation from a circular shape of $\sim 5 \mu \mathrm{m}$ in diameter to a hexagon of $\sim 8 \mu \mathrm{m}$. From which, we clearly see that all the edges with high concentration of kinks disappear quickly because of their fast propagation rates. As a result, graphene island firstly became a dodecagonal shape with six $Z Z$ and six $A C$ edges. Then, the $A C$ edges gradually became shorter and shorter because they propagate faster than $Z Z$ edges. Finally, a hexagonal graphene with six straight $Z Z$ edges was formed and the shape remained same during further evolution despite the increase of the size. The atomic details of these structures were shown in Fig. 3b. In Fig. 3c and Supplementary Fig. 5 , we present the evolutions of graphene islands with two different initial shapes during simulation. As can be clearly seen, both quickly evolved into a hexagonal graphene island with six ZZ edges. This simulation reproduced the results of kinetic Wulff construction $^{34,38}$ and presented more details of graphene shape evolution during growth and, thus, validated the KMC algorithm. Furthermore, a small size graphene island with circle shape and a $\mathrm{C}_{24}$ cluster were also simulated in Supplementary Fig. 5 . The similar process of shape evolution indicates that it is a general behavior regardless the lateral size of the island.

The lateral size of a hexagonal graphene island as a function of the simulation time is presented in Fig. $3 c$, d. It is found that the size of the graphene increases exponentially with the growth time, which implies that the island grows faster and faster or the growth rate is size dependent. This is a typical feature of nucleationmediated crystal growth, which was broadly observed during the growth of nanomaterials, such as the CVD growth of nanowires ${ }^{62}$. In our simulation, the probability of kink propagation is $\mathrm{e}^{2.2 \mathrm{eV} / \mathrm{k}_{\mathrm{B}} T} \approx 3 \times 10^{9}$ times larger than that of the nucleation on ZZ edge. On a short edge, the kink propagation time is much smaller than the nucleation time and, therefore, such a short edge has zero kink during most of its growth time and the growth rate will depend on the probability of the kink nucleation on the edge, which is proportional to the number of $Z Z$ sites of the edge or the length of the edge. As shown in $\mathrm{Sl}$, we have

$v \sim L$ or $\frac{\mathrm{d} L}{\mathrm{~d} t} \sim L$ 
(a)

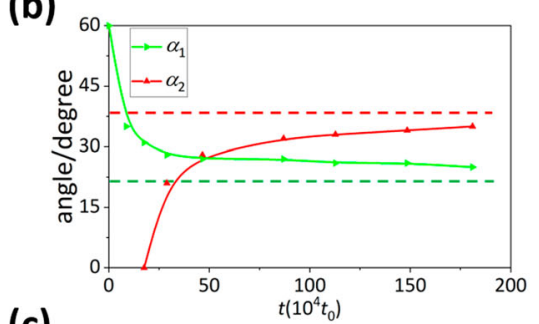

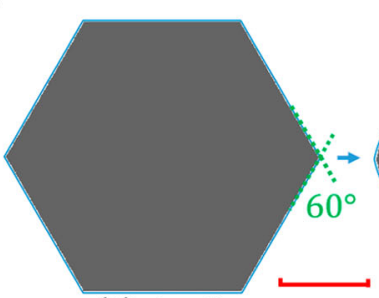

(1): $t=0$

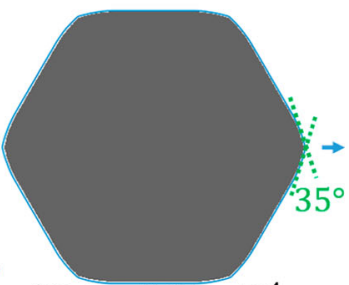

(2): $t=8.67 \times 10^{4}$

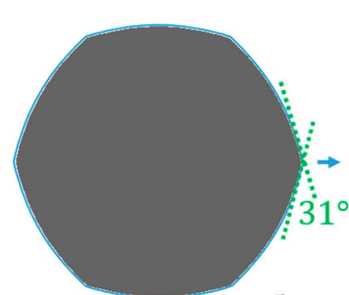

(3): $t=1.75 \times 10^{5}$

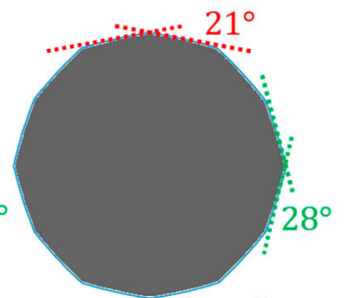

(4): $t=2.89 \times 10^{5}$

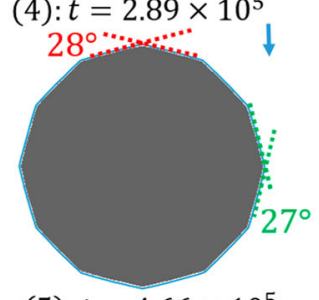
(7): $t=1.1 \times 10^{6}$
(6): $t=8.7 \times 10^{5}$
(5): $t=4.66 \times 10^{5}$

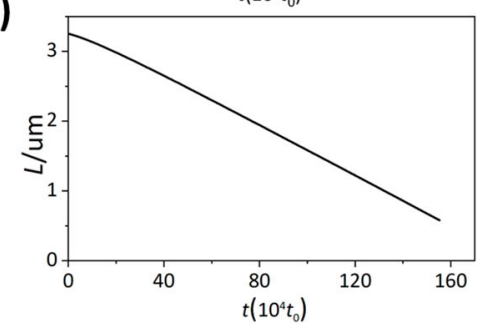

(d)
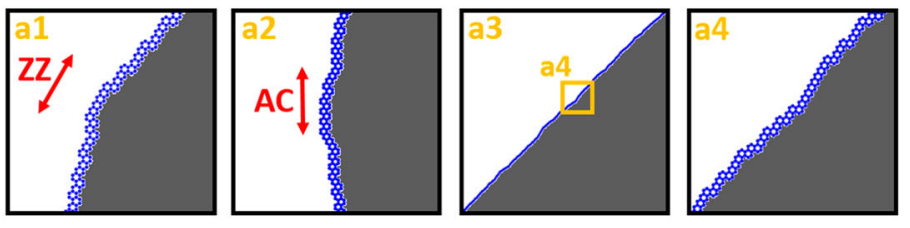

Fig. 4 Etching of a graphene island. The simulation condition is $\mu=-0.2 \mathrm{eV}$ and $k_{\mathrm{B}} T=0.1 \mathrm{eV}$. a Representative snapshots of a trajectory of KMC simulation for graphene etching. After dodecagon formed, the angles $a_{1}$ (green) and $a_{2}$ (red) are measured. The scale bar is $1 \mu \mathrm{m}$ for all snapshots. b Plot of $a_{1}$ and $a_{2}$ along with etching time. The dash lines correspond to the $19^{\circ}$ edges. c Plot of the island size in a with growth time. d Atomic details during the simulation, where a1 and a2 are corners between adjacent edges, a3 and a4 are edge with high kink concentration.

The solution of Eq. (5) is $L=\exp (a t)$, where $a=$ $0.66\left(k_{\mathrm{B}} T / h\right) \mathrm{e}^{-G_{\mathrm{b}}^{+}(2,5) / k_{\mathrm{B}} T} \approx 1600 \mathrm{~s}^{-1}$ agrees with our fitting in Supplementary Fig. 4. So far, the in situ observation of graphene CVD growth is very limited and there's no direct evidence of the edge length-dependent growth rate of graphene.

Next, we studied the etching of graphene islands on a $\mathrm{Pt}(111)$ surface. Different from graphene island growth, the fast propagating edges should survive while the slow propagating ones should disappear during an etching process ${ }^{34}$. According to Eq. (4) and the theory similar to the kinetic Wulff construction for crystal growth, the final shape of a graphene island during etching should be a dodecagon with twelve $19^{\circ}$-edges ${ }^{34}$. We performed our method in simulating the etching behaviors of graphene islands, the KMC condition was $\mu=-0.2 \mathrm{eV}$ and $k_{\mathrm{B}} T=0.1 \mathrm{eV}$. As shown in Fig. $4 a$, the evolution of a graphene island during etching starts with a regular hexagon with six ZZ edges. Firstly, the angles of the six sharp corners became larger and larger, and the six straight edges gradually became six smooth curves because the continuous kinks generation and propagation from the six sharp corners gradually reshaped the outline of the graphene island. Later, 12 straight edges along the crystallographic directions of $\sim 10.5^{\circ}$ from the six $\mathrm{ZZ}$ edges formed the circumference of the graphene island. It is surprising that the crystallographic alignments of these edges are not $19^{\circ}$ from the three $\mathrm{ZZ}$ directions as predicted by the kink nucleation and propagation theory. During further evolution, the tilt angles of the 12 edges of the circumference became larger and larger, approaching to $19^{\circ}$ gradually. We measured angles of two corners $a_{1}$ and $a_{2}$, and plotted their evolution with time in Fig. 4b. $a_{2}$ was starting from $21^{\circ}$ and gradually approaching to $38^{\circ}$, which is the angle between two $19^{\circ}$ edges. This evolution of corners has been observed in experiment ${ }^{63}$. However, the theory of kinetic Wulff construction didn't predict this observation.

The etching rate was also studied by plotting the diameter of dodecagon with the etching time in Fig. 4c. In sharp contrast to the graphene growth, it is found that the size of the island, $L$, decreases linearly with the etching time after a dodecagon formed. The linear relationship is originated from the kinetics of etching. In an etching process, most kinks nucleation events started from the corners of the islands, which is as easy as the kink propagation. Therefore, the etching rate is mainly contributed by the concentration of kinks at the edge and the whole circumference will be evolved into a shape enclosed by edges with highest kink density. This leads to a constant etching rate after a short period.

In Fig. 4d, we showed the atomic structures of the edges and corners of the dodecagonal graphene island, from which we can see that the edge is not atomically straight and, therefore the numbers of the kinks of these edges are different from that used in previous studies. So, we believe that the kink nucleation and propagation theory, which is based on the propagation of regular and straight graphene edges, is not accurate enough for the prediction of the shape evolution of graphene islands during etching or regrowth, and the atomic details can be caught by large-scale atomic simulations only.

The simulated growth and etching of a hole in graphene are presented in Fig. 5. The KMC conditions were $\mu=0.2 \mathrm{eV}$ and $k_{\mathrm{B}} T=$ $0.1 \mathrm{eV}$ for growth, and $\mu=-0.2 \mathrm{eV}$ and $k_{\mathrm{B}} T=0.1 \mathrm{eV}$ for etching. As shown in Fig. 5a, during graphene growth, the hexagonal hole starts to be filled nearby the six concave corners and the six sharp corners become rounded in large scale and, atomic details show that the angle quickly becomes larger and larger and then gradually approaching to $158^{\circ}$, the angle indicated by the theory 
(a)

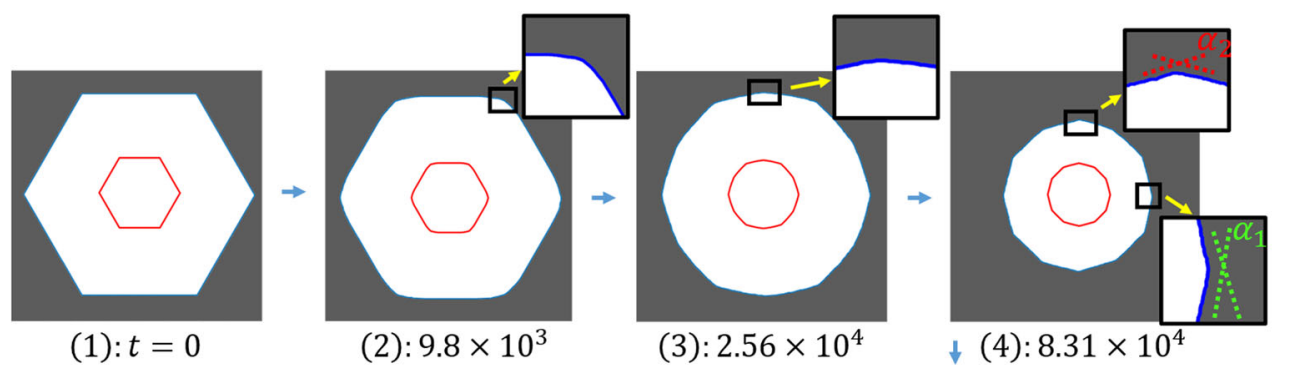

(b)

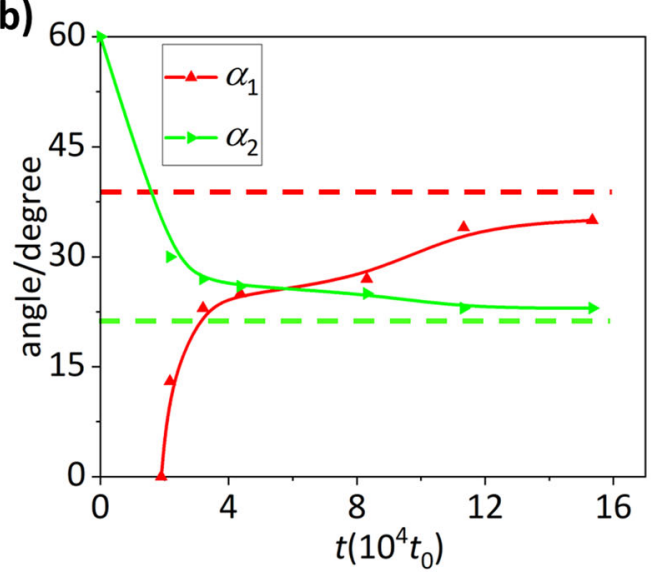

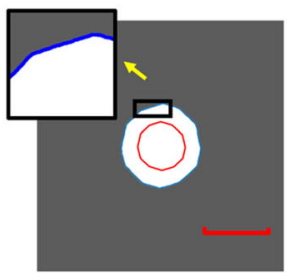

(6): $1.13 \times 10^{5}$

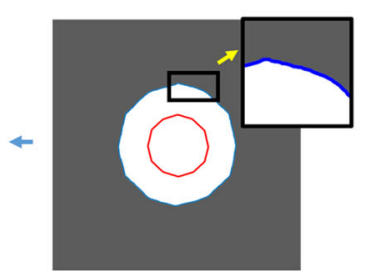

(5): $1.13 \times 10^{5}$ (c)

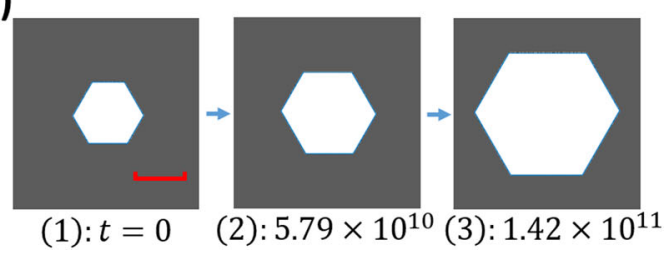

Fig. 5 Growth or etching of a hole in graphene. The KMC conditions were $\mu=0.2 \mathrm{eV}$ and $k_{\mathrm{B}} T=0.1 \mathrm{eV}$ for growth, and $\mu=-0.2 \mathrm{eV}$ and $k_{\mathrm{B}} T=$ $0.1 \mathrm{eV}$ for etching. a Growth of a hexagonal hole with all zigzag edges. The red contours are circumferences of a graphene island during etching shown in Fig. 4a, from which one can see that the hole shape evolution during growth is similar to that of island etching. The scale bar is $150 \mathrm{~nm}$ for all snapshots. b Evolution of $a_{1}$ and $a_{2}$ along with the etching time. The dash lines are corresponding angles be of dodecagon with we $19^{\circ}$ edges. c The evolution of a hole during graphene etching.

of kink nucleation and propagation. Later, another angle appears at the center of each edge and then gradually approaching to $142^{\circ}$. The evolution of two complementary angles of them is shown in Fig. 5b. In Fig. 5c, we can see that the hole keeps its original hexagonal shape with six ZZ edges during the graphene etching, which reproduces the shape evolution of graphene island during growth is very similar to that of graphene islands growth.

\section{Complementary relationship of graphene growth and etching}

To further demonstrate similarities and differences of graphene island and hole shape evolution during growth and etching, we plot the schematic diagrams of graphene and hole growth and etching in Fig. 6.

Figure $6 a$ represents the atomic structure of a graphene island and a hole near a corner of them. The straight edge of both have exactly same atomic structure, but the structures of the corners of them are complementary-the coordination number of the hexagon at the corner of a graphene island is less than that of a hexagon of a flat edge, while the coordination number of a hexagon at the corner of a hole in graphene is larger than that of a hexagon of a flat edge. So, in an etching process, removing a hexagon from a $Z Z$ edge has a smaller reaction energy than that from a concave corner of a hole (Fig. $6 \mathrm{~b}$ ). Thus, the nucleation on a ZZ edge is a threshold step of the hole evolution during etching. Such a kinetics is exactly same as the growth of hexagonal graphene island with six ZZ edges, where adding a hexagon on the convex corner is less favorable than that on a flat $\mathrm{ZZ}$ edge (Fig. $6 \mathrm{~b}$ ). So, the hole shape evolution during etching is exactly same as that of a graphene island during growth. During graphene growth, adding carbon atoms to a concave corner of a hole is energetically more favorable than that near a straight $\mathrm{ZZ}$ edge (Fig. 6c), which is similar to the etching of a graphene island, where the hexagons at the convex corners can be easily removed
(Fig. 6c). The complementary relation of graphene growth and etching is not a surprise because the hole actually becomes larger and larger during graphene etching, and smaller and smaller during growth, which is exactly opposite to the evolutions of graphene island during growth and etching.

Above KMC simulations and kinetics analysis clearly showed a fundamental principle of graphene growth and etching that the growth and etching of a graphene island and a hole in graphene are complementary, or the graphene island shape evolution during growth is same as that of hole in graphene during etching, and that of graphene etching is same as that of hole growth. Furthermore, consider the generality of the theoretical analysis, such a principle can be applied to the growth and etching of all 2D materials and even 3D materials. In is worth to note that, in ref. ${ }^{61}$, Stehle and coauthors have reported a similar behavior of hexagonal boron nitride during the growth and etching.

The complementary relationship can also be understood in macroscopic scale. In macroscopic scale, the shape evolution during growth/etching are all described by the evolution of the boundary. We considered two complementary cases, growth of a hexagonal graphene island and etching of a hexagonal hole, as example. In these two cases, the shapes of boundary are determined by the slowest propagating edges, which is the concept of kinetic Wulff construction. Since the initial shape of boundaries and the slowest edge are both same, the evolution route would be exactly same in two cases.

In summary, we introduced a large-scale KMC method to simulate the growth and etching of graphene island, and holes in graphene. The KMC algorithm allows use to perform largescale simulations to understand the evolution of graphene up to 10-micrometers in sizes without losing atomic details. Benefited by the large-scale simulation, we have revealed a complementary relationship of graphene growth and etching 
(a) Graphene
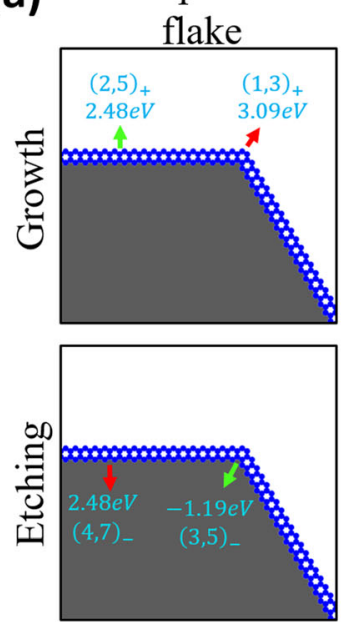

(b)
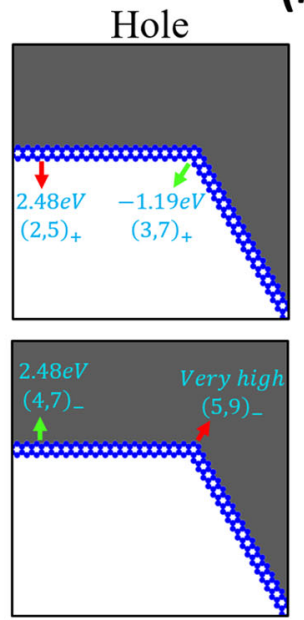

Flake

growth:

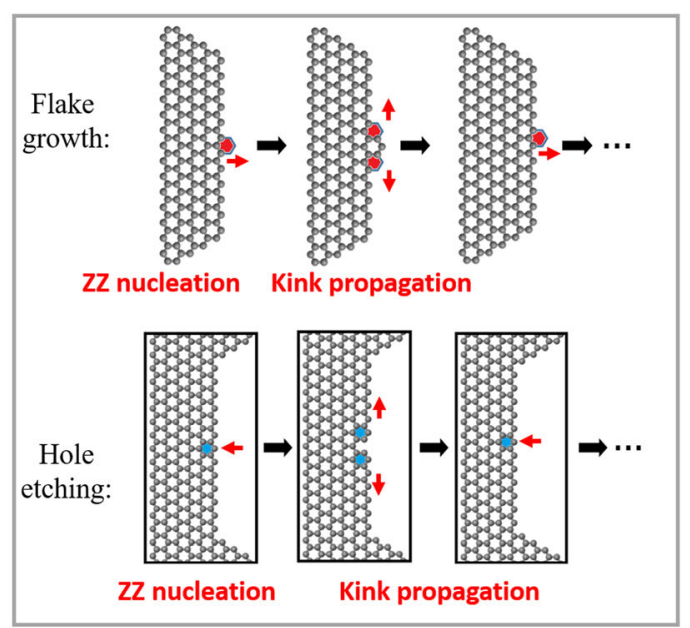

Z nucleation Kink propagation

ZZ nucleation

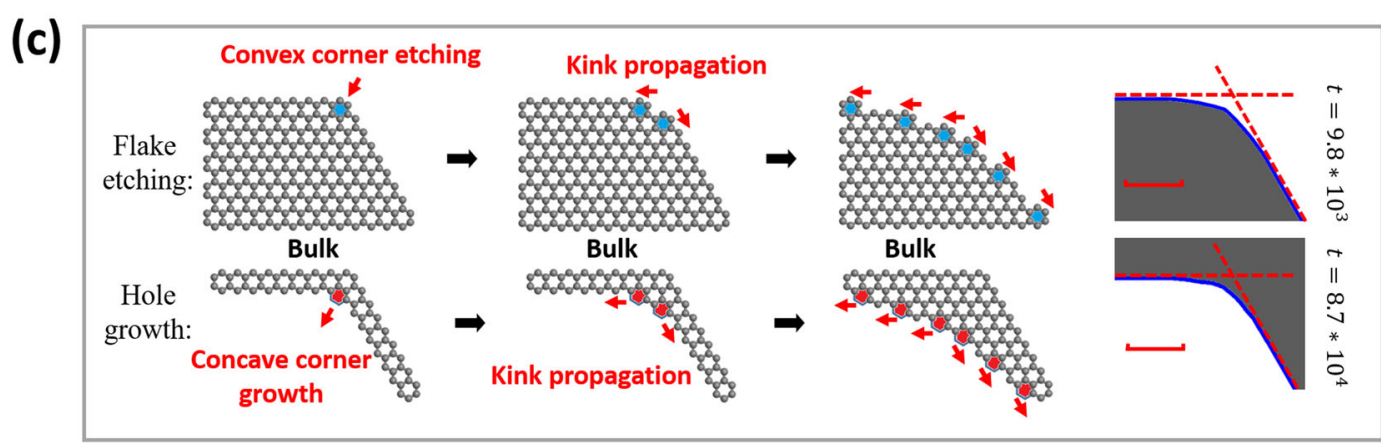

Fig. 6 Schematic illustration of the mechanism of the growth and etching of graphene island and hole, respectively. a Geometric structure of corners of graphene island and hole and the reaction energies of corresponding events during growth and etching. The events pointed by green arrows are energetically more favorable than those pointed by red arrows. b Similarity between graphene island growth and hole etching. c Similarity between graphene island etching and hole growth, and two snapshots taken from KMC simulations of island etching and hole growth, the scale bars are $1 \mu \mathrm{m}$ and $150 \mathrm{~nm}$, respectively.

-that the shape evolution of a graphene island during growth is exactly same as that of a hole during etching, and that of a graphene island during etching is exactly same as that of a hole during growth. Such a complementary relationship can be easily extended to understand the growth and etching of other 2D and 3D materials, which provide a theoretical basis to understand the relationship of materials growth and etching.

\section{METHODS}

\section{First-principle calculations}

The reaction energies of key events are obtained by using first-principle calculations, the atomic models are introduced in the Supplementary Materials.

The Vienna ab initio simulation package is employed for all first-principle calculations $^{64}$. The Perdew-Burke-Ernzerhof parameterization of generalized gradient approximation (GGA) is used for the exchange-correlation function ${ }^{65}$. The projector-augmented wave potentials are used for ion-electron interactions ${ }^{66}$. The DFT-D2 VDWs density functional is used for the corrections of the interlayer VDWs interaction ${ }^{67}$. The electronic selfconsistency criterion is set to $10^{-4} \mathrm{eV}$. For geometry relaxation, the force on atoms is converged below $0.01 \mathrm{eV} \AA^{-1}$. A vacuum layer of $15 \AA$ is used. The substrate contains three layers atoms with bottom two layers fixed. For graphene bulk energy on substrates, all calculations are sampled by $4 \times$ $4 \times 1 k$-mesh. For step energy calculation, all the calculations are sampled with $1 \times 1 \times 1 k$-point.

\section{DATA AVAILABILITY}

All data generated and/or analyzed during this study are included in this article and its Supplementary Information file. The raw simulation data and the other datasets are available from the author on reasonable request.

\section{CODE AVAILABILITY}

The results were simulated by using a code developed by us. We will consider releasing the code later and the usage of this code requires signing an agreement with the code developer.

Received: 2 June 2020; Accepted: 16 December 2020; Published online: 26 January 2021

\section{REFERENCES}

1. Novoselov, K. S. et al. Electric field effect in atomically thin carbon films. Science 306, 666-669 (2004).

2. Lee, C. et al. Measurement of the elastic properties and intrinsic strength of monolayer graphene. Science 321, 385-388 (2008).

3. Neto, A. H. C. et al. The electronic properties of graphene. Rev. Mod. Phys. 81, 109-162 (2009).

4. Sarma, S. D. et al. Electronic transport in two-dimensional graphene. Rev. Mod. Phys. 83, 407-470 (2011).

5. Mortazavi, B. Ultra-high stiffness and thermal conductivity of graphene like C3N. Carbon 118, 25-34 (2017).

6. Dean, C. R. et al. Boron nitride substrates for high-quality graphene electronics Nat. Nanotechnol. 5, 722-726 (2010). 
7. Hernandez, Y. et al. High-yield production of graphene by liquid-phase exfoliation of graphite. Nat. Nanotechnol. 3, 563-568 (2008).

8. Nicolosi, V. et al. Liquid exfoliation of layered materials. Science 340, 1226419 (2013).

9. Yi, M. \& Shen, Z. A review on mechanical exfoliation for the scalable production of graphene. J. Mater. Chem. A 3, 11700-11715 (2015).

10. Parvez, K. et al. Exfoliation of graphite into graphene in aqueous solutions of inorganic salts. J. Am. Chem. Soc. 136, 6083-6091 (2014).

11. Hackley, J. et al. Graphitic carbon growth on $\mathrm{Si}(111)$ using solid source molecular beam epitaxy. Appl. Phys. Lett. 95, 133114 (2009).

12. Sutter, P. W., Flege, J. I. \& Sutter, E. A. Epitaxial graphene on ruthenium. Nat. Mater. 7, 406-411 (2008).

13. Berger, $C$. et al. Electronic confinement and coherence in patterned epitaxial graphene. Science 312, 1191-1196 (2006).

14. Virojanadara, C. et al. Homogeneous large-area graphene layer growth on $6 \mathrm{HSiC}$ (0001). Phys. Rev. B 78, 245403 (2008).

15. Emtsev, K. V. et al. Towards wafer-size graphene layers by atmospheric pressure graphitization of silicon carbide. Nat. Mater. 8, 203-207 (2009).

16. Riedl, C. et al. Quasi-free-standing epitaxial graphene on SiC obtained by hydrogen intercalation. Phys. Rev. Lett. 103, 246804 (2009).

17. Kim, K. S. et al. Large-scale pattern growth of graphene films for stretchable transparent electrodes. Nature 457, 706-710 (2009).

18. Huang, M. et al. Highly oriented monolayer graphene grown on a $\mathrm{Cu} / \mathrm{Ni}$ (111) alloy foil. ACS Nano 12, 6117-6127 (2018).

19. Roberto, M. \& Cristina, G.-A. Review of CVD synthesis of graphene. Chem. Vap. Depos. 19, 297-322 (2013).

20. Murdock, A. T. et al. Controlling the orientation, edge geometry, and thickness of chemical vapor deposition graphene. ACS Nano 7, 1351-1359 (2013).

21. Weber, N.-E. et al. Metal-free synthesis of nanocrystalline graphene on insulating substrates by carbon dioxide-assisted chemical vapor deposition. Carbon 112, 201-207 (2017).

22. Wan, X. et al. Towards wafer-size strictly monolayer graphene on copper via cyclic atmospheric chemical vapor deposition. Carbon 110, 384-389 (2016).

23. Li, X. et al. Large-area synthesis of high-quality and uniform graphene films on copper foils. Science 324, 1312-1314 (2009).

24. Obraztsov, A. N. Chemical vapour deposition: making graphene on a large scale. Nat. Nanotechnol. 4, 212-213 (2009).

25. Malesevic, A. et al. Synthesis of few-layer graphene via microwave plasmaenhanced chemical vapour deposition. Nanotechnol 19, 305604 (2008).

26. Chen, Z. et al. Three-dimensional flexible and conductive interconnected graphene networks grown by chemical vapour deposition. Nat. Mater. 10, 424-428 (2011).

27. Mattevi, C., Kim, H. \& Chhowalla, M. A review of chemical vapour deposition of graphene on copper. J. Mater. Chem. 21, 3324-3334 (2011).

28. $\mathrm{Wu}, \mathrm{T}$. et al. Fast growth of inch-sized single-crystalline graphene from a controlled single nucleus on Cu-Ni alloys. Nat. Mater. 15, 43-47 (2016).

29. $\mathrm{Xu}, \mathrm{X}$. et al. Ultrafast epitaxial growth of metre-sized single-crystal graphene on industrial Cu foil. Sci. Bull. 62, 1074-1080 (2017).

30. $\mathrm{Yu}, \mathrm{Q}$. et al. Control and characterization of individual grains and grain boundaries in graphene grown by chemical vapour deposition. Nat. Mater. 10, 443-449 (2011).

31. Vlassiouk, l. et al. Role of hydrogen in chemical vapor deposition growth of large single-crystal graphene. ACS Nano 5, 6069-6076 (2011).

32. Wu, B. et al. Self-organized graphene crystal patterns. NPG Asia Mater. 5, e36 (2013).

33. Geng, D. et al. Uniform hexagonal graphene islands and films grown on liquid copper surface. Proc. Natl Acad. Sci. USA 109, 7992-7996 (2012).

34. $\mathrm{Ma}, \mathrm{T}$. et al. Edge-controlled growth and kinetics of single-crystal graphene domains by chemical vapor deposition. Proc. Natl Acad. Sci. USA 110, 20386-20391 (2013).

35. Dong, J., Zhang, L. \& Ding, F. Kinetics of graphene and 2D materials growth. Adv. Mater. 31, 1801583 (2019).

36. Hao, Y. et al. The role of surface oxygen in the growth of large single-crystal graphene on copper. Science 342, 720-723 (2013).

37. $\mathrm{Xu}, \mathrm{X}$. et al. Ultrafast growth of single-crystal graphene assisted by a continuous oxygen supply. Nat. Nanotechnol. 11, 930-935 (2016).

38. Artyukhov, V. I., Liu, Y. \& Yakobson, B. I. Equilibrium at the edge and atomistic mechanisms of graphene growth. Proc. Natl Acad. Sci. USA 109, 15136-15140 (2012).

39. Gao, J. et al. Graphene nucleation on transition metal surface: structure transformation and role of the metal step edge. J. Am. Chem. Soc. 133, 5009-5015 (2011).

40. Yuan, Q. et al. Magic carbon clusters in the chemical vapor deposition growth of graphene. J. Am. Chem. Soc. 134, 2970-2975 (2012).
41. Shu, H. et al. Edge structural stability and kinetics of graphene chemical vapor deposition growth. ACS Nano 6, 3243-3250 (2012).

42. Zhang, X. et al. Role of hydrogen in graphene chemical vapor deposition growth on a copper surface. J. Am. Chem. Soc. 136, 3040-3047 (2014).

43. Zhang, X. et al. How the orientation of graphene is determined during chemical vapor deposition growth. J. Phys. Chem. Lett. 3, 2822-2827 (2012).

44. Gao, J. \& Ding, F. The structure and stability of magic carbon clusters observed in graphene chemical vapor deposition growth on Ru (0001) and Rh (111) surfaces. Angew. Chem. Int. Ed. 53, 14031-14035 (2014).

45. Gao, J. et al. Formation of carbon clusters in the initial stage of chemical vapor deposition graphene growth on Ni (111) surface. J. Phys. Chem. C 115, 17695-17703 (2011).

46. Robertson, J. Heterogeneous catalysis model of growth mechanisms of carbon nanotubes, graphene and silicon nanowires. J. Mater. Chem. 22, 19858-19862 (2012).

47. Zhang, W. et al. First-principles thermodynamics of graphene growth on $\mathrm{Cu}$ surfaces. J. Phys. Chem. C 115, 17782-17787 (2011).

48. Shu, H., Tao, X.-M. \& Ding, F. What are the active carbon species during graphene chemical vapor deposition growth? Nanoscale 7, 1627-1634 (2015).

49. Zhuang, J. et al. Morphology evolution of graphene during chemical vapor deposition growth: a phase-field theory simulation. J. Phys. Chem. C 123, 9902-9908 (2019).

50. Meca, E. et al. Epitaxial graphene growth and shape dynamics on copper: phasefield modeling and experiments. Nano Lett. 13, 5692-5697 (2013).

51. Laturia, A., Van de Put, M. L. \& Vandenberghe, W. G. Dielectric properties of hexagonal boron nitride and transition metal dichalcogenides: from monolayer to bulk. npj 2D Mater. Appl. 2, 1-7 (2018).

52. Momeni, K. et al. Multiscale computational understanding and growth of $2 \mathrm{D}$ materials: a review. npj Comput. Mater. 6, 1-18 (2020).

53. Meng, L. et al. Molecular dynamics simulation of chemical vapor deposition graphene growth on Ni (111) surface. J. Phys. Chem. C 116, 6097-6102 (2012).

54. Jiang, H. \& Hou, Z. Large-scale epitaxial growth kinetics of graphene: a kinetic Monte Carlo study. J. Chem. Phys. 143, 084109 (2015).

55. Chen, S. et al. A kinetic Monte Carlo model for the growth and etching of graphene during chemical vapor deposition. Carbon 146, 399-405 (2019).

56. Chen, S. et al. An all-atom kinetic Monte Carlo model for chemical vapor deposition growth of graphene on Cu (1 111 1 $)$ substrate. J. Phys. Condens. Matter 32, 155401 (2020).

57. Taioli, S. Computational study of graphene growth on copper by first-principles and kinetic Monte Carlo calculations. J. Mol. Model. 20, 2260 (2014).

58. Wang, D. et al. Thermodynamics and kinetics of graphene growth on $\mathrm{Ni}(111)$ and the origin of triangular shaped graphene islands. J. Phys. Chem. C 122, 3334-3340 (2018).

59. Dahal, A. \& Batzill, M. Graphene-nickel interfaces: a review. Nanoscale 6, 2548-2562 (2014).

60. Zhu, L. \& Ding, F. How the moiré superstructure determines the formation of highly stable graphene quantum dots on Ru (0001) surface. Nanoscale Horiz. 4, 625-633 (2019).

61. Rao, R. et al. In situ evidence for chirality-dependent growth rates of individual carbon nanotubes. Nat. Mater. 11, 213-216 (2012).

62. Kashchiev, D. Dependence of the growth rate of nanowires on the nanowire diameter. Cryst. Growth Des. 6, 54-1156 (2006).

63. Stehle, Y. Y. et al. Anisotropic etching of hexagonal boron nitride and graphene: question of edge terminations. Nano Lett. 17, 7306-7314 (2017).

64. Kresse, G. \& Furthmüller, J. Efficient iterative schemes for ab initio total-energy calculations using a plane-wave basis set. Phys. Rev. B 54, 11169 (1996).

65. Perdew, J. P., Burke, K. \& Ernzerhof, M. Generalized gradient approximation made simple. Phys. Rev. Lett. 77, 3865 (1996).

66. Blöchl, P. E. Projector augmented-wave method. Phys. Rev. B 50.24, 17953 (1994).

67. Grimme, S. Semiempirical GGA-type density functional constructed with a longrange dispersion correction. J. Comput. Chem. 27, 1787-1799 (2006).

\section{ACKNOWLEDGEMENTS}

X.K. and F.D. acknowledges support from the Institute for Basic Science (IBS-R019-D1) of South Korea and the computational resources from CMCM, IBS. L.Z. is supported by support from NSFC (Grant No. 11704141) and Natural Science Foundation of the Jiangsu Higher Education Institutions of China (Grant No. 18KJA140001).

\section{AUTHOR CONTRIBUTIONS}

F.D. conceived the research. X.K., J.Z., and L.Z. carried out the code and theoretic simulations. X.K. and F.D. wrote the manuscript. All authors discussed the results and commented on the work. 


\section{COMPETING INTERESTS}

The authors declare no competing interests.

\section{ADDITIONAL INFORMATION}

Supplementary information is available for this paper at https://doi.org/10.1038/ s41524-020-00489-y.

Correspondence and requests for materials should be addressed to F.D.

Reprints and permission information is available at http://www.nature.com/reprints

Publisher's note Springer Nature remains neutral with regard to jurisdictional claims in published maps and institutional affiliations.
Open Access This article is licensed under a Creative Commons Attribution 4.0 International License, which permits use, sharing, adaptation, distribution and reproduction in any medium or format, as long as you give appropriate credit to the original author(s) and the source, provide a link to the Creative Commons license, and indicate if changes were made. The images or other third party material in this article are included in the article's Creative Commons license, unless indicated otherwise in a credit line to the material. If material is not included in the article's Creative Commons license and your intended use is not permitted by statutory regulation or exceeds the permitted use, you will need to obtain permission directly from the copyright holder. To view a copy of this license, visit http://creativecommons. org/licenses/by/4.0/.

(c) The Author(s) 2021 